\title{
A Qualitative Investigation into the Lived Experiences of Critical Care Nurses Working on Night Shifts
}

\author{
Halmat Authman Rasheed; Department of Nursing, College of Nursing, Hawler Medical University, Erbil, Iraq. \\ (Correspondence: halmat.rasheed@hmu.edu.krd)
}

Newroz Ghazi Aziz; Department of Nursing, College of Nursing, Hawler Medical University, Erbil, Iraq. Goran Aboobaker Osman; Department of Nursing, College of Nursing, Hawler Medical University, Erbil, Iraq.

\section{ABSTRACT}

Background and objectives: Shift work is used in the hospitals and residential treatment centres to provide patients with continuous health care. During their night shift, nurses are often faced with different health problems either due to the stressful work environment or because of the sophisticated instruments to be utilized in intensive care units. The present study was aimed at investigating the lived experience of nurses working on the night shifts at Rizgary Teaching Hospital located in Erbil, Iraqi Kurdistan Region.

Methods: A qualitative phenomenological study was conducted to describe the lived experiences of nurses working on night shifts. In doing so and in order to collect the required data, open-ended semi-structured in-depth interviews were carried out to explore thoughts, feelings, and lived experiences of 15 nurses who were working on the night shifts in the Intensive Care Unit (ICU) at Rizgary Teaching Hospital in Erbil. The collected data were then analyzed by Van Manen's (1990) method.

Results: Study participants were between 26 and 38 years old. The majority were male (60\%) and 40\% were female. Regarding marital status, 10 participants were married and 5 were single. Most of the nurses had Bachelor degrees (86.6\%). The respondents work experience was between 3 and 8 years. Almost all of the participating nurses had similar lived experiences during their night shifts. The nurses' lived experiences gave way to the emergence of four themes namely workload, psychosocial issues, sleep disturbance and physiological issues.

Conclusion: The present study showed that nurses working on night shifts faced many problems and issues such as poor sleep quality sleep, working for a long time, fatigue and anxiety, back pain, mood disturbance and lack of concentration.

Keywords: Nurses, Critical care unit, Qualitative research, Interview, Lived experience

\section{INTRODUCTION}

Due to the nature of health care and given the emergencies that may arise, nurses who are a major part of the healthcare team are often required to work at any time of the day or night to provide continuing care to patients. In doing so, nurses usually suffer from different health problems either due to the stressful work environment or because of the sophisticated equipment used in the intensive care unit [1]. Despite the importance of these health problems and their effect on the quality of health care delivered to patients, few studies have focused on 
the nurses' lived experiences during the night shift and the effects on their day-today routine activities. It is stated that female nurses who work night shifts experience more issues and difficulties, especially if they have responsibilities in their family [2]. Results of relevant research studies have shown that working the night shift can have a negative impact on sleep, social life, physical and mental health, family life, job-related stresses, and job performance [3].As a result of shift rotation, extended work schedules, and prolonged contact with irritable and depressed patients, nurses are exposed to psychological stress, which may emerge as taking on extra work, showing emotional withdrawal, engaging in substance abuse, or exhibiting depression [4]. Therefore, and to deliver healthcare services of acceptable quality, nurse managers need to monitor nursing personnel regarding such behaviors, confront inefficient nurses whose poor performance leads to a decline in productivity, and refer them to appropriate support groups and or medical care [5]. As shown by results of relevant research studies, nurses working in certain specialities and departments such as intensive care units (ICU) are more likely to experience psychological stress because ICUs are characterized by the presence of intricate machinery, high noise level, physical contact with blood, vomitus and infection hazards, continual time pressure, and unpredictable emergencies [6].Shift working schedules have been studied in few studies whose results indicated that the quality of health care, patient satisfaction, treatment duration, absenteeism at work, the effectiveness of healthcare measures, and productivity can be seriously influenced by a poorly designed working schedule [7]. Therefore, healthcare and hospital authorities need to take different factors into account while designing the nurses' working schedules.
In general, medical and nursing services cannot be delivered well without assigning nurses to work in night shift; however, working the night shift can have serious effects on the nurses' personal and working life, and as a result the quality of the delivered healthcare services and the patients and nurses' satisfaction drop dramatically. In this regard, it is necessary to consider the lived experiences of nursing personnel working night shifts to design working schedules in a way that quality of healthcare services and patient and nurse satisfaction rise [8]. The present qualitative study was carried out to investigate the lived experience of nurses working the night shift at Rizgary Teaching Hospital located in Erbil, Iraqi Kurdistan Region.

\section{METHODS}

Since the present study was aimed at investigating the lived experience of the nurses working the night shift in ICU, which is a complicated phenomenon due to a particular cultural and religious background of the target population, i.e. Kurdish nurses living in the Iraqi Kurdistan Region, a phenomenological method was employed. Participants: 15 nurses ( 9 male and 6 female) working night shifts in the ICU of Rizgary Teaching Hospital of Erbil, Iraqi Kurdistan Region participated in the study. Each of the nurses worked two days per week, 12 hours per shift, and they had approximately 4 years of work experience in intensive care units. A purposive sampling method was used to investigate their lived experiences of working night shifts. Data collection: It is suggested that data collection in qualitative studies should be carried out through semi-structured interviews allowing the interviewer to control the pace, style, and question order to elicit the interviewee's fullest responses and help the interviewee answer the research questions in their own way [9].Generally, 
qualitative methods through in-depth interviews are employed to gather information about the lived experience of individuals, and because the individuals' culture, the system of values, standards and expectations shape their lived experience, a phenomenological method was utilized in the present study. Phenomenology is aimed at clarifying the meaning, nature, quality, and context of the individuals' experiences of a unique issue [10], and semistructured in-depth interviews were used to investigate the thoughts, feelings, and lived experiences of nurses working on the night shift. Data were collected from 1st of March, 2020 to 1st of April, 2020. Before the interviews, a list of main questions aimed at scaffolding the interviews, keeping the questioning on the topic, and linking what was asked in individual interviews to the overall design was devised. During the interviews, as suggested by research, some probing questions such as (tell me more about that, how did that make you feel, how will this affect you.... etc.) were asked in relation to the subject area [11]. In addition, open-ended questions were used in the in-depth interviews to obtain more insight into the issue under investigation. Moreover, to come up with a deeper understanding of the lived experiences, feelings, and thoughts of the nurses, a friendly relationship was established with the nurses [10].Interviews, each of which lasted 30 minutes, were held at the nurses' home. Following the nurses' consent, the interviews were recorded and then transcribed verbatim. The transcripts were proofread by both the nurses and the researcher to see if there would be any mistakes or not. Nurses were given unique codes (as Nurse No. 1 , No. 2, etc.), which were used in the transcripts, since the responses given by the interviewees may be affected if the anonymity is not assured. The participants were assured that their information would be completely confidential [12].Data analysis: In order to investigate the lived experiences of the nurses working the night shifts at Rizgary Teaching Hospital, the six methodological steps proposed by VanManen's method (1990) were utilized to analyze the collected data. To analyze qualitative data and reach a high level of abstraction, as suggested by research studies, hermeneutic approaches such as the one proposed by VanManen are commonly utilized [13]. The 6 methodological steps proposed in this method include "Turning to the nature of lived experience", "Investigating experience as we live it", "Reflecting on the essential themes which characterize the phenomenon", "Describing the phenomenon in the art of writing and rewriting", "Maintaining a strong and orientated relation to the phenomenon", and "Balancing the research context by considering the parts and the whole" [14].Lincoln and Guba's fourcriterion gold standard consisting of credibility, dependability, conformability, and transferability was used to maintain the rigour of the study [15]. Moreover, to ensure the credibility of the findings, we kept a prolonged engagement with the data, two researchers were involved in the analysis and interpretation of data. Different sources of the same information, such as numerous interviews, were used to validate the findings.[16]. The dependability and confirmability of the findings were assured by an academic auditor. The auditor checked whether the analysis was in line with accepted standards and examined the analysis process and records for accuracy. Ethical ConsiderationsAfter the required permission was obtained from the hospital president, the necessary approval was gained from the Ethics Committee of College of Nursing, Hawler Medical University (HMU) No. (4) on 29 January 2020. Before the data collection, informed consent was 


\section{RESULTS}

Participants were between 27 and 38 years old. The majority of them were male (60\%) and $40 \%$ were female. Regarding marital status, 10 of the participants were married and 5 of the respondents were single.
Most of the nurses had Bachelor degrees (86.6\%). The respondents work experience was between 4 and 8 years. Four main themes with sub-themes were identified: workload, psychosocial issues, sleep disturbance, and physiological issues.

Table 1: Characterization of interviewees by sex, age, Marital status, education and work experience.

\begin{tabular}{|c|c|c|c|c|c|}
\hline SN & Sex & Age & Marital status & Educational level & Work experience/years \\
\hline 1 & Male & 32 & Married & Bachelor degree & 6 \\
\hline 2 & Female & 30 & Marie & Bachelor Degree & 4 \\
\hline 3 & Male & 29 & Single & Bachelor degree & 4 \\
\hline 4 & Male & 38 & Married & Bachelor degree & 9 \\
\hline 5 & Female & 30 & Married & Bachelor degree & 4 \\
\hline 6 & Male & 32 & Married & Bachelor degree & 7 \\
\hline 7 & Female & 29 & Single & Diploma degree & 4 \\
\hline 8 & Male & 31 & Married & Bachelor degree & 5 \\
\hline 9 & Male & 33 & Married & Bachelor degree & 4 \\
\hline 10 & Female & 35 & Married & Diploma degree & 8 \\
\hline 11 & Male & 28 & Single & Bachelor degree & 4 \\
\hline 12 & Female & 27 & Single & Bachelor degree & 4 \\
\hline 13 & Female & 32 & Married & Bachelor degree & 5 \\
\hline 14 & Male & 30 & Single & Bachelor degree & 5 \\
\hline 15 & Male & 36 & Married & Bachelor degree & 8 \\
\hline
\end{tabular}


Workload The analysis of the transcripts of the in-depth interviews indicated, the nurses believed that work demand in the night shift depends on the department. They stated that the workload during the day and night shifts do not differ significantly in some departments. Working hours in both day and night shifts are 12 hours, which is quite long. They thought; however, work demand during the night shift is heavier because there are fewer nurses to shoulder the workload. In this regard, Nurse No. 3 said: "When it comes to the work demand during night and day shift, there is no significant difference, because both shifts consist of 12 hours of work, and we are in shortage of nurses during the night shift. Some departments are busy during day and night shift."

Nurse No. 13:"...night shifts are a bit strenuous because there are usually fewer nurses compared to day shifts... nurses who work the night shifts need to work as a team because of the nurse shortage..."

As stated above, this nurse believes that if the nurses working on night shifts form a team and work together, the workload can decrease, which leads to their better performance and as a result an increase in the quality of the delivered healthcare services. Lack of sufficient nursing staff during the night shifts, which leads to an increase in workload, was also mentioned by Nurse 7 who said: "It's not about longer working hours, the working hours are equal to those of day shift, both are 12 hours, but most of the time we have to work alone, and there are not enough nursing or medical staff to help and take a part of the responsibility. As a result, the workload during the night shift is felt as heavier." The same issue was referred to by Nurse No. 9 who said, "If there are more nurses in the night shift, the work demand will be the same as day shift." Psychosocial issues The results of the present study indicated that the nurses had to deal with numerous difficulties and problems due to the requirements of the ICU ward where they have to be highly alert and have the ability to make critical, often life or death decisions, which was interpreted as psychosocial issues. It was observed that working on the night shift caused the nurses to experience fatigue, which might in turn result in personal and family issues, including mood disturbances, decreased performance, and negative effects on personal relationships with colleagues, if it is not dealt with appropriately. The night shift also caused the nurses to experience stress and anxiety, such that they interpreted the night shift as strenuous due to the lack of nursing staff and the resulting increased workload. In this regard, Nurse No. 5 said: "As nurses, we encounter several challenges during the night shift. The challenges that we face include exhaustion, anxiety, stress, and poor social relationships because of the responsibilities that we have at home. These challenges are attributed to the inefficiency of the nursing staff..." Most of the nurses pointed out that their social status within the community could influence their adaptation to and attitude towards night shifts. For example, the nurse's marital status, family needs, social values, and lifestyle within the community can influence her attitude and feelings towards working at night. In this regard, Nurse No. 2 said: "I've got a 2-year-old daughter, and I have to ask my mother or sister to take care of her whenever I work in night shift, which automatically makes me hate the night shift, and it also affects my performance during the night shift because I'm always thinking of my daughter and worried about her." The nurses also thought that the night shifts disturb the balance between the desired lifestyle and work. Women play a major role in domestic life, and they are expected to undertake domestic chores 
such as taking care of their children and doing family chores. In this regard, Nurse No. 10, a 35-year-old woman, said: "Most of the time, I leave my family and provide care to patients during the night shift which usually starts at $7 \mathrm{pm}$ and finishes at $7 \mathrm{am}$. Because of my career, I have to get someone to attend to my children and do my housework. I am married and would like to have the company of my husband, but because of work issues, social relationships are affected in some ways because I cannot accompany my husband when he attends social events" Social and familial issues affect working the night shift and vice versa. In this regard, Nurse No. 6 said, "Workload of working in night shift makes me stressed out, which in turn influences my social and family life." Sleep disturbance Sleep was referred to by the nurses as the main function disturbed by working the night shifts. Nurses who work night shifts have to sleep during the day and when the surrounding conditions (light, noise, and temperature) are not desirable, their sleep will be disturbed and its duration drops. Working the night shift can also affect sleep quality because sleeping during the day is light and more distracted compared to night sleep, which greatly affects the nurses' performance at work during their shift. In this regard, Nurse No. 4, a 38year-old male nurse, said: "Poor quality of sleep at night is the problem that I encounter regarding the sleep when working the night shift; the sleep duration becomes too short. Our sleep during the day is affected by our surroundings such as noise and light. To fall asleep, I usually go to bed early, read novels, or drink beverages. This helps me to sleep especially during the first day when I need to work the night shift" Nurse No. 8 also referred to sleep problems and said: "The day before my night shift, I cannot usually sleep well, because everywhere is so light, and there is a lot of noise from outside, so I feel sleepy at work all the time, and I'm sure it has a negative effect on what I do at work" Moreover, lack of concentration during night shifts because of lack of sleep was mentioned as one of the challenges those night shift nurses encountered. In this regard, Nurse No. 15 said, "ICU nurses need to be quite alert and pay attention to everything, but I cannot concentrate well during my night shifts, because I usually feel sleepy." Nurse No. 1 also had the same problem and said, "Because I cannot sleep well during the day, my performance is negatively affected during night shifts." Health issues Nurses who work on night shifts usually experience health issues ranging from the lack of concentration as a result of workload and lack of sleep during their night shifts to physiological hazards, headache, persistent tiredness, backache, and even abortion because they have to stand for hours, lift heavy things, walk a lot, and make lots of decisions. Nurse No. 12, a 27-year-old female nurse, described the night shift as more strenuous and tiring and stated that nurses working in night shifts are more likely to undergo occupational injuries such as pricks and muscular strains. She said: "Health issues that I face include headache, persistent tiredness, backache due to long hours of standing, and lack of sleep. Most of the time, we also face or have heard that nurses complain about their occupational injuries such as needle pricks and muscular strains due to too much workload and lack of concentration." Nurse No. 11 also stated, "I experience fatigue and feel tired during and after my night shifts due to lack of breaks and workload." Moreover, Nurse No. 14 referred to her health problems caused by working night shifts and said, "In addition to sleep problems and lack of concentration, I sometimes cannot stand on my feet because of my backache." 


\section{DISCUSSION}

The results of the present study showed that the nurses interpreted their lived experience of working night shifts as extra workload, psychosocial issues, sleep problems, and physiological issues. Data analysis indicated that there was an extra workload on nurses during their night shifts, although they worked the same 12 hours as day shifts, because, as nurses mentioned, there were fewer nurses during night shifts, and they had to deliver healthcare services to patients alone. This finding is in line with those of the study conducted by Moattari \& Jahromi in 2013) [17], who referred to the fact that nurses undergo excessive working pressure, which is an organizational issue and needs to be resolved by the hospital management by increasing the number of nurses working night shifts. This finding is also in agreement with the study done by Abdullah Karim et al. (2017) [18]. However, Nasrabadi et al. (2009) [19], stated that lack of nursing staff can have some advantages for nurses. Firstly, they have to do most of the tasks by themselves, which makes them more skillful, and secondly, they need to establish friendly relationships with most patients, which in turn affects their social life and working performance positively. Another theme emerging out of the nurses' lived experience was psychological issues which included personal and family issues such as mood disturbances, decreased performance, negative effects on personal relationships with colleagues and family members, and imbalance between the desired lifestyle and work. This finding is in agreement with those of the studies carried out by Costa (2016) [20] and Moattari and Jahromi (2013) [17], who declared that night shift nurses in family-oriented communities are faced with numerous familial and social issues which might be resolved by making or changing the public culture and ideology about night-shift working nurses through the mass media. The third theme that emerged out of the nurses' lived experience was sleep disturbance, which as participants claimed, caused the work performance and concentration at work to drop significantly. This finding is also in agreement with the study done by Zion and Shochat (2019) [21], who stated that taking short naps during night shifts, is considered as an effective way to lower levels of sleepiness and improve nurses' psychomotor performance. The last theme of the present study was physiological issues including stress, headache, persistent tiredness, backache, and even abortion. Insomnia, fatigue, and stomachache are common physiological problems that night-shift working nurses are faced with. This problem might be resolved by increasing the number of nurses attending night shifts so that the workload drops significantly, and the nurses will not have to stand and work all their shifts long, which in turn causes such health problems to improve [22].Limitation of the study took place only in one hospital (Rizgary Teaching Hospital), and it would be better to include nurses from different hospitals and also from different cities. Participants could be asked why they chose the night shift to work in, which was not asked by the researcher.

\section{CONCLUSION}

The nurses in the present study faced many problems and issues during their night shift work, such as poor quality of sleep, working for a long time without rest, fatigue and anxiety, back pain, mood disturbance and lack of concentration, which in turn affect the quality of their performance. 


\section{RECOMMENDATION}

[6] Vahedian-Azimi A, Hajiesmaeili M, Kangas-

It is recommended that the number of nurses in each night shift should be reviewed by the hospital administration and nurses could cooperate during their night to provide patients with healthcare services of better quality and to minimize psychosocial and physiological problems among the nursing staff.

\section{CONFLICT OF INTEREST AND FUNDING OF THE STUDY}

There is no conflict of interest and funding source.

\section{REFERENCES.}

[1] Chappel SE, Verswijveren SJ, Aisbett B, Considine J, Ridgers ND. Nurses' occupational physical activity levels: A systematic review. International Journal of Nursing Studies. 2017; 73: 52-62.https://doi.org/ https://doi.org/10.1016/

j.ijnurstu.2017.05.006.

[2] Saksvik IB, Bjorvatn B, Hetland H, Sandal $\mathrm{GM}$, Pallesen S. Individual differences in tolerance to shift work - A systematic review. Sleep Medicine Reviews. 2011; 15(4): 221-35.https://doi.org/https:// doi.org/10.1016/j.smrv.2010.07.002.

[3] Cheng WJ, Cheng Y. Night shift and rotating shift in association with sleep problems, burnout and minor mental disorder in male and female employees. Occupational and Environmental Medicine. 2017; 74(7): 483-88. https://doi.org/10.1136/ oemed-2016-103898.

[4] Shiffer D, Minonzio M, Dipaola F, Bertola $M$, Zamuner AR, Dalla Vecchia, et al. Effects of Clockwise and Counterclockwise Job Shift Work Rotation on Sleep and Work-Life Balance on Hospital Nurses. International Journal of Environmental Research and Public Health. 2018; 15(9): 2038.https://doi.org/10.3390/ ijerph15092038.

[5] Raffenaud A, Unruh L, Fottler M, Liu AX, Andrews D. A comparative analysis of work-family conflict among staff, managerial, and executive nurses. Nursing Outlook.2020; 68(2): 231-41. https:// doi.org/https://doi.org/10.1016/ j.outlook.2019.08.003. niemi $M$, Fornés-Vives J, Hunsucker RL, Rahimibashar F, et al. Effects of Stress on Critical Care Nurses: A National CrossSectional Study. Journal of Intensive Care Medicine. 2019; 34(4): 311-22. https:// doi.org/10.1177/0885066617696853.

[7] Redeker NS, Caruso CC, Hashmi SD, Mullington JM, Grandner M, Morgenthaler TI. Workplace interventions to promote sleep health and an alert, healthy workforce. Journal of Clinical Sleep Medicine. 2019; 15(4): 649-57. https://doi.org/10.5664/jcsm.7734.

[8] Vidotti V, Ribeiro RP, Galdino MJ, Martins JT. Burnout syndrome and shift work among the nursing staff. Revista Latino-Americana de Enfermagem. 2018; 26(0). https:// doi.org/10.1590/1518-8345.2550.3022

[9] Devadas B. A Critical Review of Qualitative Research Methods in Evaluating Nursing Curriculum Models: Implication for Nursing Education in the Arab World. Journal of Education and Practice. 2016; 7(7): 119-26.

[10] Burns M, Peacock S. Interpretive phenomenological methodologists in nursing: A critical analysis and comparison. Nursing Inquiry. 2019; 26(2): 1-10. https://doi.org/10.1111/ nin. 12280 .

[11] Au A. Thinking about cross-cultural differences in qualitative interviewing: Practices for more responsive and trusting encounters. Qualitative Report. 2019; 24(1): 58-77.

[12] Johnson JL, Adkins D, Chauvin S. A review of the quality indicators of rigor in qualitative research. American Journal of Pharmaceutical Education. 2020; 84(1): 138-46. https:// doi.org/10.5688/ajpe7120

[13] Saleh MY, Al-Amer R, Al Ashram SR, Dawani $H$, Randall S. Exploring the lived experience of Jordanian male nurses: A phenomenological study. Nursing Outlook. 2020; 68(3): 313 -23.https://doi.org/https://doi.org/10.1016/ j.outlook.2019.10.007

[14] Imani B, Mohamad Khan Kermanshahi S, Vanaki Z, Kazemnejad Lili A, Zoghipaydar MR. Iranian Hospital Nurses' Lived Experiences of Emotional Intelligence: A Phenomenological Study. Issues in Mental Health Nursing. 2019; 40(8): 712-19. https:// doi.org/10.1080/01612840.2017.1395497

[15] Nikbakht Nasrabadi A, Mohammadpour A, Fathi M. A New Horizon in Life: Experiences of Patients Receiving Chemotherapy. Global Journal of Health Science. 2016; 8(4): 10208.https://doi.org/10.5539/gjhs.v8n4p102. 
[16] Faghihi SA, Khankeh HR, Hosseini SJ, Arabshahi SK, Faghih Z, Parikh SV, et al. Improving continuing medical education by enhancing interactivity: lessons from Iran. Journal of Advances in Medical Education \& Professionalism. 2016; 4(2), p.54.

[17] Moattari M, Jahromi MF. Novice Nurses' Perception of Working Night Shifts: A Qualitative Study. Journal of Caring Sciences.2013;2(3):169-76.https:// doi.org/10.5681/jcs.2013.021.

[18] Abdulla Karim M, Rafii F, Nikbakht Nasrabadi A, Kurdistan Nurses' Feelings and Experiences About Patients' Death in ICUs: A Case Study in Kurdistan Region, Iraq. Journal of Client-Centered Nursing Care. 2017; 3(1): 3-10.

[19] Nasrabadi AN, Rasoolzadeh N, Emami A, Seif $\mathrm{H}$, Latifi M. Night shift work experiences among Iranian nurses: A qualitative study: Original Article. International Nursing Review. 2009; 56(4): 498-503. https:// doi.org/10.1111/j.1466-

7657.2009.00747.x.

[20] Costa G. No Title. In: Iskra-Golec I, BarnesFarrell J. ed. Social and Family Issues in Shift Work and Non-Standard Working Hours. Springer, Cham; 2016: 19-35. https://doi.org/https:// doi.org/10.1007/978-3-319-42286-2_2.

[21] Zion N, Shochat T. Let them sleep: The effects of a scheduled nap during the night shift on sleepiness and cognition in hospital nurses. Journal of Advanced Nursing. 2019; 75(11): 2603-615. https:// doi.org/10.1111/jan.14031.

[22] Mahmoued TM, Mohamed N, Saleh A, Ali N. Night Shift Work and Its Impact on Health Status of Nurses. Zagazig Nursing Journal. 2017; 13(2): 53-66. 